\title{
Early VLF perturbations observed in association with elves
}

\author{
Á. Mika ${ }^{1}$, C. Haldoupis ${ }^{1}$, T. Neubert ${ }^{2}$, H. T. Su ${ }^{3}$, R. R. Hsu ${ }^{3}$, R. J. Steiner ${ }^{1}$, and R. A. Marshall ${ }^{4}$ \\ ${ }^{1}$ Department of Physics, University of Crete, Heraklion, Greece \\ ${ }^{2}$ Danish National Space Center, Copenhagen, Denmark \\ ${ }^{3}$ Department of Physics, National Cheng Kung University, Tainan, Taiwan \\ ${ }^{4}$ Space, Telecommunications, and Radioscience Laboratory, Stanford University, Stanford, USA
}

Received: 23 March 2006 - Revised: 5 July 2006 - Accepted: 19 July 2006 - Published: 13 September 2006

\begin{abstract}
VLF remote sensing is used to detect lowerionospheric electron density changes associated with a certain type of transient luminous events known as elves. Both ground- and satellite-based observations of elves are analysed in relation to VLF data acquired at various receiver sites in Europe, the United States and Antarctica. Ground-based observations were performed during the EuroSprite2003 campaign, when five elves were captured by low-light cameras located in the Pyrenees. Analysis of VLF recordings from Crete shows early VLF perturbations accompanying all of the elves. A large dataset consisting of elves captured by the ISUAL (Imager of Sprites and Upper Atmospheric Lightning) payload on Taiwan's FORMOSAT-2 satellite over Europe and North America has also been analysed. Early/fast VLF perturbations were found to accompany some of the elves observed over Europe. However, no VLF perturbations were detected in relation to the elves observed by ISUAL over North America. The present analysis - based on the largest database of optical elve observations used for VLF studies so far - constitutes evidence of processes initiated by the lightning EMP (electromagnetic pulse) causing electron density changes in the lower ionosphere in line with theoretical predictions. It also proves that sub-ionospheric electron density changes associated with elves can intrude to lower heights and thus perturb VLF transmissions. The possibility of VLF detection, however, depends on several factors, e.g., the distance of the elve from the receiver and the transmitterreceiver great circle path (GCP), the altitude of the ionised region and the characteristics of the VLF transmitter, as well as the EMP energy, which occasionally may be sufficient to cause optical emissions but not ionisation.

Keywords: Ionosphere (Ionisation mechanisms; Ionosphereatmosphere interactions; Ionospheric disturbances).
\end{abstract}

Correspondence to: Á. Mika

(agnes@physics.uoc.gr)

\section{Introduction}

Electromagnetic pulses (EMPs) released by lightning discharges into the lower ionosphere can lead to momentary optical emissions called elves (e.g., see reviews by Rowland, 1998; Rodger, 1999). These phenomena, appearing at altitudes between 75 and $105 \mathrm{~km}$, constitute a certain type of transient luminous events (TLEs) in the upper atmosphere. Other forms of TLEs are red sprites and blue jets. Elves consist of a rapidly expanding ring of luminosity reaching lateral extents of about $500 \mathrm{~km}$, with their occurrence depending on the strength of the causative discharge irrespective of its polarity (Barrington-Leigh and Inan, 1999). Elves are visible for about $1 \mathrm{~ms}$, which makes their detection from the ground rather difficult. They can be captured from space more frequently due to the much lower atmospheric attenuation rates (Chern et al., 2003).

Theoretical models indicate that in addition to optical emissions, strong lightning EMPs can also lead to ionisation in the lower ionosphere (Taranenko et al., 1993; Rowland, 1998). At elve-altitudes this ionisation is expected to be lasting for many minutes (e.g., Rodger et al., 2001) and may affect very low frequency (VLF) radio wave propagation inside the Earth-ionosphere waveguide. For instance, the name "elves" was originally introduced by Fukunishi et al. (1996) as an acronym for "Emissions of Light and VLF perturbations due to EMP Sources", but no examples of accompanying VLF perturbations were given by the authors. Although there are several VLF studies carried out during transient luminous event activity in the upper atmosphere, it has not been conclusively shown yet that elves can lead to VLF perturbations (Rodger, 2003).

The most common perturbations on sub-ionospheric VLF transmissions caused by direct lightning effects on the ionosphere are the "early/fast" events, characterised by an abrupt onset followed by slower relaxation times of several tens of seconds. Early/fast VLF signatures are believed to be caused by forward scattering from localised regions of ionisation enhancements above an active thunderstorm close to the great

Published by Copernicus GmbH on behalf of the European Geosciences Union. 
circle path (GCP) between the VLF transmitter and the receiver (e.g., see Inan et al., 1988, 1993). It is now believed that sprites in the lower ionosphere associate closely with early/fast VLF perturbations, although these perturbations may not be unique to them. This relation was hinted by early measurements over the United States, reported by Inan et al. (1996b) and Dowden et al. (1996), while more recent observations in Europe, reported by Haldoupis et al. (2004) and Mika et al. (2005), suggested a nearly one-to-one relationship.

The issue of VLF perturbations occurring in association with elves has received little attention so far. There exist a few conference presentations (Reising et al., 1995; Takahashi et al., 1995; Dowden et al., 1995), as cited by Strangeways (1996) and Fukunishi et al. (1996), but no refereed publications dedicated to the topic. Recently, Mende et al. (2005) presented indirect evidence of electron density enhancements in relation to an elve event observed by the ISUAL (Imager of Sprites and Upper Atmospheric Lightning) instrument on board the Taiwanese FORMOSAT-2 (former ROCSAT-2) satellite. The average electron density was estimated to be 210 electrons $\mathrm{cm}^{-3}$ over a circular region with a diameter of $\sim 165 \mathrm{~km}$ at $\sim 90 \mathrm{~km}$ altitude having an assumed height extent of $10 \mathrm{~km}$.

The scope of the present study is to report on the presence or not of elve-related VLF perturbations, and it was motivated by an early/fast event presented by Mika et al. (2005), which accompanied an elve recorded during the EuroSprite2003 campaign (for details on EuroSprite2003 see Neubert et al., 2005). In order to extend the range of experimental evidence available on elve-related VLF perturbations both ground- and space-based optical observations were used. Five elve events identified during EuroSprite2003 are considered in our analysis in addition to 68 elves captured by ISUAL over Europe and North America. This constitutes the largest database of optical elve recordings used for VLF studies so far. VLF data used for comparisons with the optical observations were obtained with Stanford University VLF receivers located in Greece, the United States and Antarctica.

\section{Electron density changes induced by the lightning EMP in the lower ionosphere}

Elves are initiated by the impact on the lower ionosphere of the electromagnetic pulse emitted during a strong lightning return stroke in the troposphere (e.g., see Taranenko et al., 1993; Rowland et al., 1996; Barrington-Leigh and Inan, 1999). According to models (Rowland, 1998), for a vertical discharge the emitted EMP intensity has a minimum above the discharge and a maximum in a ring around it, affecting a doughnut-shaped region. Given the short EMP duration (typically $\sim 50-100 \mu \mathrm{s}$ ), the atmospheric electrical conductivity can not shield the electric field before it reaches the ionosphere. As a result, the maximum absorption of wave energy (i.e. the maximum electron heating) occurs at $80-95 \mathrm{~km}$ altitudes, where the leading edge of the EMP reflecting from the bottom of the ionosphere constructively interferes with the trailing part of the pulse (Taranenko et al., 1993). The first positive band of $\mathrm{N}_{2}$ is excited in this altitude range by the impact of electrons with energies $>7 \mathrm{eV}$ (Inan et al., 1996a), leading to optical emissions produced in a volume with the shape of a doughnut with a thickness of $\sim 30 \mathrm{~km}$, expanding to at least $\sim 200-700 \mathrm{~km}$ laterally (Barrington-Leigh and Inan, 1999), and lasting for $\sim 400 \mu \mathrm{s}$.

Impact ionisation of $\mathrm{N}_{2}$, having an electron energy threshold of $15.6 \mathrm{eV}$, can also be caused by a single EMP at 80-95 km altitudes, leading to electron density enhancements from a few to a few tens of per cent (Taranenko, 1993). Successive EMPs can cause a density increase of many hundreds of per cent of the ambient values (Taranenko et al., 1993; Rodger et al., 2001), affecting a large area of $\sim 3 \times 10^{5} \mathrm{~km}^{2}$ (Barrington-Leigh and Inan, 1999). At lower altitudes, extending down to $\sim 80 \mathrm{~km}$, where electrons are only mildly heated, dissociative attachment to molecular oxygen $\left(\mathrm{O}_{2}+\mathrm{e} \rightarrow \mathrm{O}+\mathrm{O}^{-}\right)$dominates, leading to decreases in the electron density of several per cent (Inan et al., 1996a; Sukhorukov et al., 1996).

The anticipated electron density enhancements due to ionisation and the depletions caused by dissociative attachment lead to a sharpening of the electron density profile, which, if located below the nighttime VLF reflection height $(\sim 80-90 \mathrm{~km})$, might perturb the propagation of subionospheric VLF transmissions. If this happens, then based on theoretical grounds, the large, smoothly varying ionised region produced during elves is expected to cause forward scattering of the incident VLF transmitter signals (Dowden, 1996). This would lead to step-like early VLF perturbations having long relaxation times due to the long lifetime of electrons at these altitudes. For instance, for a doubling of the electron density at $90 \mathrm{~km}$ altitude it takes $\sim 30 \mathrm{~min}$ to return to within 10\% of the ambient value (Rodger et al., 2001).

The only papers published on elve-related VLF perturbations so far are those of Hobara et al. $(2001,2003)$ and Mika et al. (2005). Hobara et al. (2001, 2003) reported perturbations in association with 7 elves out of a total of 17 . However, there is some doubt if these perturbations were indeed caused by ionisation changes relating to elves. The only figure included in the paper shows a RORD (Rapid Onset Rapid Decay) event, which is most likely caused by heating effects only, and not by changes in the electron density (Inan et al., 1996b). On the other hand, Mika et al. (2005) observed an early/fast type VLF perturbation in association with an optical elve captured during the EuroSprite2003 campaign, which constitutes indirect evidence for ionisation changes in relation to elves. An overview of this event is given in the next section. The only other evidence on ionisation production during an elve event has recently been provided by Mende et al. (2005), based on multiwavelength photometric observations on board the FORMOSAT-2 satellite. 


\section{Observational results}

In the following, the results of the search for early VLF perturbations in relation to elves are summarised. First, five elve events captured during the EuroSprite 2003 campaign are presented and discussed. Second, the largest dataset of elves used for VLF studies so far, collected by ISUAL, is analysed. For the elves seen above Europe VLF data were supplied by VLF receivers located in Greece and France, while for those observed over North America data were obtained from various VLF receivers located in the United States and Antarctica.

\subsection{EuroSprite2003 elves}

During the two month duration of EuroSprite2003 (for details on this campaign see Neubert et al., 2005) six elves were captured by the low-light cameras. VLF data were available for five events, from receivers located on the Island of Crete and in Nançay, France.

Optical observations of elves were made from the Pic du Midi Observatory (OMP) in the French Pyrenees. An automated camera system was used, which consisted of two unintensified low-light CCD cameras mounted on a motorised pan-tilt unit. This allowed observations from $-35^{\circ}$ to $+35^{\circ}$ elevation and $360^{\circ}$ in azimuth with a field-of-view of $22.5^{\circ}$. The exposure time of the individual images was $20 \mathrm{~ms}$ and the image timestamp was correct to within $12 \mathrm{~ms}$.

At the Crete receiver site the broadband signal is detected by a $1.7 \times 1.7 \mathrm{~m}^{2}$ magnetic square-loop antenna and bandpass filtered to a range of 9 to $45 \mathrm{kHz}$ with $100 \mathrm{kHz}$ sampling frequency using GPS timing. Amplitude and phase time series of six VLF transmitter signals are extracted from the broadband data and recorded with both $20 \mathrm{~ms}$ and $1 \mathrm{~s}$ resolution, from 17:00 to 05:00 UTC. The transmitters monitored at Crete during EuroSprite2003 were the following: HWV $(18.3 \mathrm{kHz}$, Le Blanc, France), HWU $(20.9 \mathrm{kHz}$, Rosnay, France), GQD (22.1 kHz, Anthorn, UK), DHO (23.4 kHz, Rhauderfehn, Germany), NRK (37.5 kHz, Keflavik, Iceland), and NAU ( $40.75 \mathrm{kHz}$, Aguada, Puerto Rico).

The Nançay VLF station is a two-channel (north-south and east-west) broadband $(\sim 350 \mathrm{~Hz}-50 \mathrm{kHz})$ receiver using two right-isosceles triangular antennas with $4.2 \mathrm{~m}$ height and $8.4 \mathrm{~m}$ base, mounted on a central pole. The sampling rate for both channels is $100 \mathrm{kHz}$. During the campaign the receiver was operated daily from 21:00 to 03:00 UTC.

Information on the location and peak current intensity of cloud-to-ground (CG) lightning discharges was obtained by Météorage, the French national lightning detection system. According to Morel and Sénési (2000), the detection efficiency of Météorage is better than $90 \%$ and it provides the time of occurrence of CG discharges with $1 \mathrm{~ms}$ accuracy and their geographic location with $\sim 4 \mathrm{~km}$ precision.

One of the elve events, captured during a storm over central France on July 22, 2003, has already been presented by
Mika et al. (2005). It was accompanied by an early/fast VLF perturbation identified in the HWV - Crete signal. The perturbation had a magnitude of $\sim 0.4 \mathrm{~dB}$ and a relaxation time of $\sim 2 \mathrm{~min}$, comparable to those observed in relation to red sprites captured during EuroSprite2003 (Mika et al., 2005). According to the French lightning detection system there was no cloud-to-ground discharge coincident with the occurrence of the elve. The closest discharge in time was a $+\mathrm{CG}$ with a peak current of $119 \mathrm{kA}$, preceding the elve by $\sim 400 \mathrm{~ms}$. However, broadband VLF data recorded at Nançay revealed the existence of a very strong sferic coincident with the occurrence of the elve, the causative lightning discharge of which was apparently missed by Météorage.

During the night of 23-24 July 2003 four elves were recorded by the low-light cameras at Pic du Midi. The locations of the cloud-to-ground discharges recorded by Météorage for a $\pm 5 \mathrm{~min}$ interval around the elves are presented in Fig. 1. Also shown are the GCPs of the transmitter signals on which early VLF perturbations were observed. According to Météorage, three of the elves were accompanied by strong positive CG discharges having peak current intensities of 217, 110 and $119 \mathrm{kA}$ respectively, all originating from the storm centre located close to the HWV - Crete GCP, whereas for the fourth elve only a much weaker, $-30.3 \mathrm{kA}$ CG flash was identified. Broadband VLF data recorded at Nançay, however, revealed a dominant sferic due to a much stronger discharge in addition to the $-30.3 \mathrm{kA}$ one, which most likely caused the elve. Examination of the raw data from the individual Météorage sensors showed that there was a strong positive cloud-to-ground discharge occurring at 00:29:12.752 UTC, following the $-30.3 \mathrm{kA}$ one by only $6 \mathrm{~ms}$. This discharge, however, was only detected by two of the sensors, which was inadequate for triangulation and thus it was excluded from the Météorage event list.

In order to determine the geographic position of the elve two independent methods were combined. First, the direction of the VLF energy source for the elve-related sferic was estimated using broadband Nançay data. Second, star fields identified in the optical image taken from Pic du Midi were used to find the azimuth of the center of the elve. Then the intersection of these two directions gave the location of the elve. In order to find the direction from which the VLF energy emanates, the signals received by the two antennas at Nançay (orientated in the magnetic north-south and east-west directions, respectively) were combined. Due to the presence of noise, a signal threshold was introduced, below which the values were ignored. Then the data was collected in bins with a width of $5^{\circ}$, and the number of data points per bin was counted. The results are presented in the top panel of Fig. 2, whereas the bottom panel shows the combined intensity of the broadband signals. The elve occurred in the time frame marked by the two blue vertical lines, which account for the $20 \mathrm{~ms}$ frame integration time and an uncertainty in timing of $\pm 12 \mathrm{~ms}$. The time series also revealed a small sferic associated with the $-30.3 \mathrm{kA}$ discharge recorded by Météorage, 


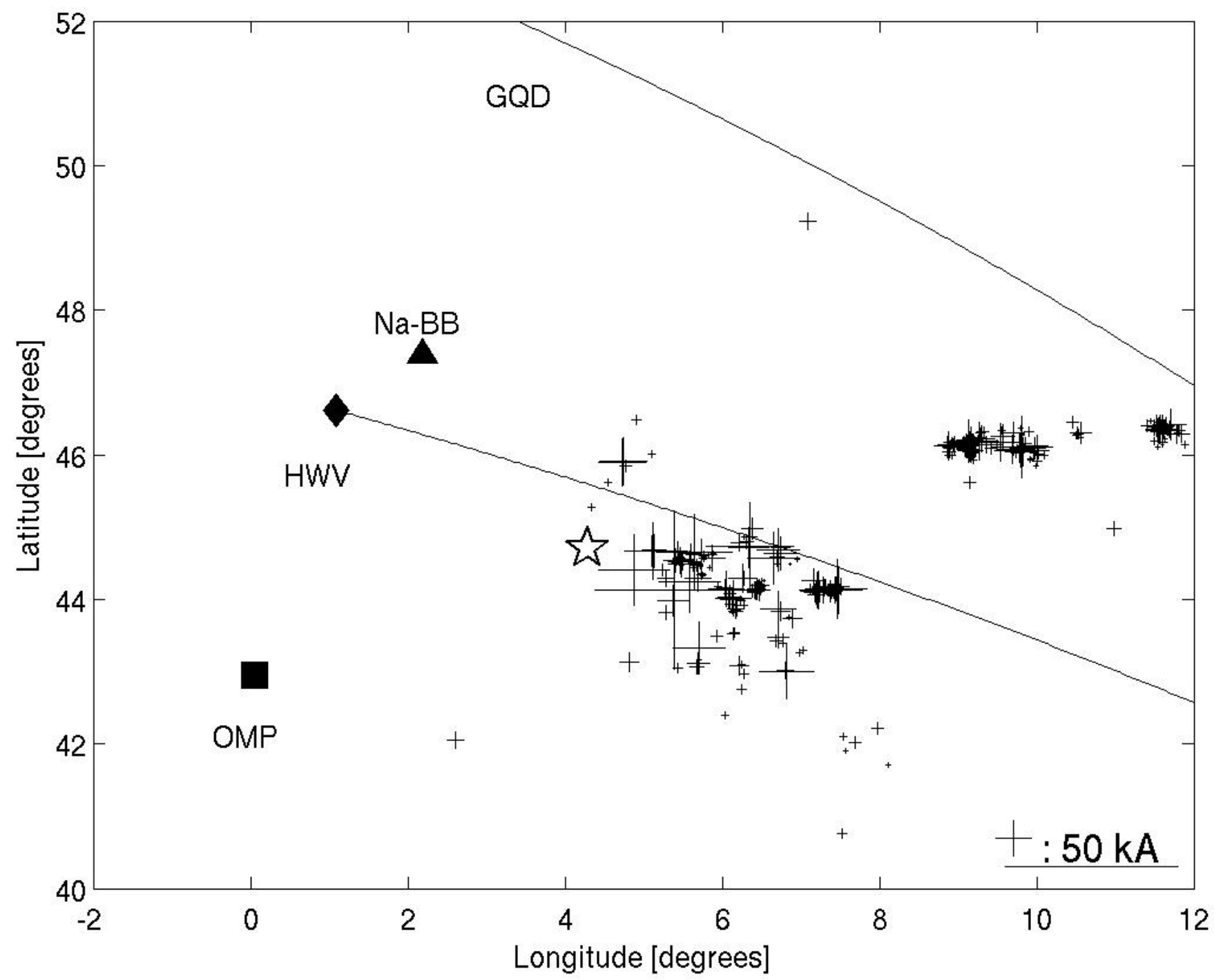

Fig. 1. Locations of CG discharges (crosses) observed within a \pm 5 min interval around the elve occurrences during the night of $23-24$ July 2003. The sizes of the crosses are scaled with the peak current intensities. The star marks the location of an elve, estimated by other methods, for which lightning data was unavailable (discussed in chapter 3.1). The locations of the Pic du Midi Observatory and the Nançay broadband VLF receiver are also shown along with the GCPs of the transmitter signals to Crete on which early VLF perturbations were identified.

followed by a much stronger peak after $6 \mathrm{~ms}$. The location of the causative discharge of the latter sferic, which most probably caused the elve, failed to be identified by the lightning detection network. The results of this analysis are illustrated in Fig. 2 which shows that the highest number of counts occurred at $\sim-45^{\circ}$ angle, measured from the east, which pointed towards the main storm. The other method was to infer the azimuth of the elve with the help of the star fields identified in the optical image. For this purpose the "Cartes du Ciel" program, freely available on the Internet, was used (http://www.stargazing.net/astropc/index.html), which gave an angle of $\sim 65^{\circ}$ from the north to the centre of the elve seen from Pic du Midi. The intersection of the two direc- tions, marked by a star in Fig. 1, positioned the elve inside the storm close to the HWV - Crete GCP.

Early VLF perturbations were observed at Crete in association with all four elves captured during the night of 23-24 July 2003. They were identified in the HWV and GQD transmitter signals. The perturbation magnitudes varied from 0.15 to $0.3 \mathrm{~dB}$ and the onset durations (i.e. the time needed for the maximum perturbation to be reached) were in the range of $20 \mathrm{~ms}-2 \mathrm{~s}$, including both early/fast and early/slow (e.g., see Haldoupis et al., 2004) type events, while the relaxation times were $2-3 \mathrm{~min}$. Example perturbations accompanying the elve event discussed previously in Fig. 2 are presented in Fig. 3 with the elve image also shown in the top panel. 


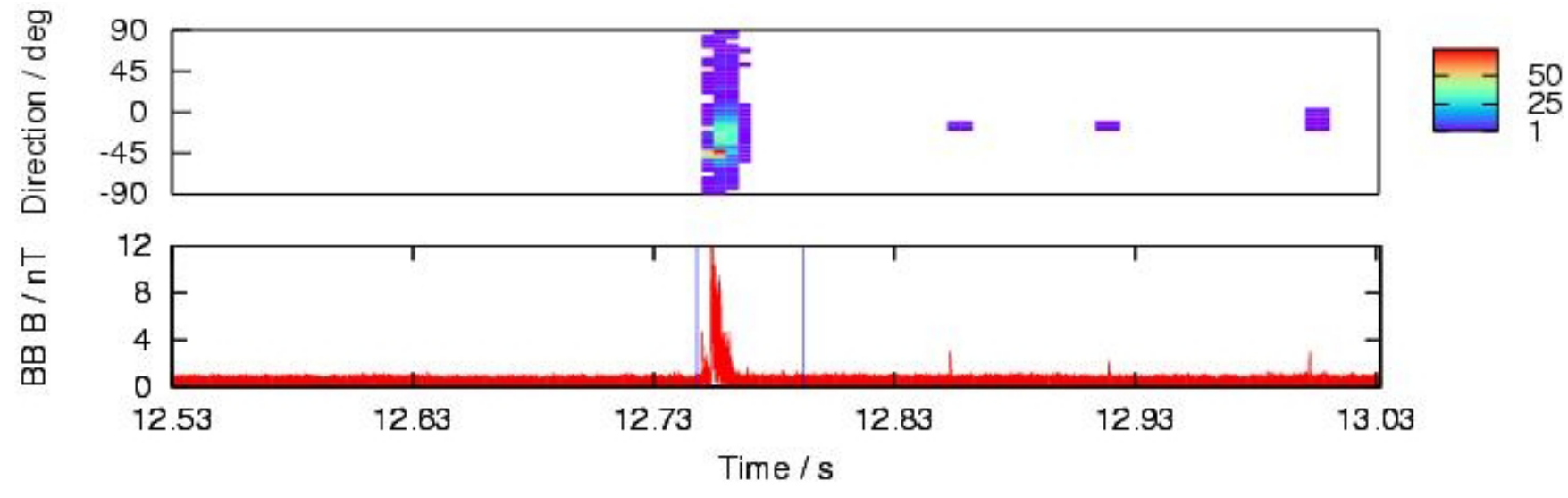

Fig. 2. The lower panel shows the combined signal intensity of the broadband EW and NS VLF recordings made at Nançay $\pm 250 \mathrm{~ms}$ around the elve occurring on 24 July 2003 at 00:29:12.757 UTC. The blue vertical lines mark the occurrence of the elve accounting for the $20 \mathrm{~ms}$ frame integration time and a timing uncertainty of $\pm 12 \mathrm{~ms}$. The top panel shows the direction of arrival of the sferic signal at about $\sim-45^{\circ}$ measured from the east.

The elve-producing discharges were all located at $\sim 1950 \mathrm{~km}$ from the Crete receiver, close to the HWV - Crete GCP, but off the GQD link at lateral distances $\sim 640 \mathrm{~km}$ (Fig. 1). This suggests that the early VLF perturbations seen at Crete were most likely caused by narrow angle forward scattering, in line with theoretical expectations for the scattering pattern of the broad and smoothly varying ionised region which is anticipated to be produced by a lighting EMP (Dowden, 1996).

\subsection{Elves captured by ISUAL}

The Imager of Sprites and Upper Atmospheric Lightning (ISUAL) is a scientific payload on board the Taiwanese FORMOSAT-2 satellite, aiming at providing the first global survey of TLEs by taking advantage of the smaller atmospheric attenuation rates as compared to ground observations. FORMOSAT- 2 is operating in a $891 \mathrm{~km}$ altitude sunsynchronous orbit. During the night the overfly of the satellite is around 10 p.m. local time which, with the imager pointing sideways, covers the region around local midnight (Chern et al., 2003). The field of view of ISUAL is $\sim 20^{\circ}$ in the horizontal and $\sim 5^{\circ}$ in the vertical, which gives an observed area of about $1024 \mathrm{~km}^{2}$. ISUAL is equipped with a six-channel spectrophotometer, a two-channel array photometer and a visible light intensified CCD imager with a filter wheel, all mounted on a common platform and bore-sighted in the same direction. The imager has a programmable frame rate of $1 \mathrm{~ms}$ to $1 \mathrm{~s}$. It is operated continuously during the night pass, but data are saved only when given trigger criteria are met.

During the period October, 2004-March, 200518 elves were observed by ISUAL over Europe. VLF data was available for 17 events from the Crete receiver site. The transmitters monitored during this time were: HWV $(18.3 \mathrm{kHz}$,
Le Blanc, France), GBZ (19.6kHz, Criggion, UK), UMS (20.5 kHz, Nizniy Novgorod, Russia), HWU (20.9 kHz, Rosnay, France), NAA $(24.0 \mathrm{kHz}$, Cutler, Maine, USA), and NRK $(37.5 \mathrm{kHz}$, Keflavik, Iceland). In Fig. 4 the locations of the elve-producing lighting discharges (marked by circles), along with the GCPs from the different transmitters to the Crete receiver are presented. Eight elves were situated at lateral distances less than $\sim 300 \mathrm{~km}$ from the closest GCP, while the rest were located between $\sim 550$ and $1150 \mathrm{~km}$. Six VLF perturbations were observed on multiple links in association with three elves (marked by filled circles on Fig. 4), all located close to the GCPs. These distances, measured from the elve locations to the observing GCPs, were less than $500 \mathrm{~km}$ in all cases, while the distances to the Crete receiver were $\sim 400-950 \mathrm{~km}$. All perturbations were of the early/fast type, their onsets being coincident with elves within the time resolution of the measurements and having onset durations $<50 \mathrm{~ms}$. They were identified only in the amplitude time series and had magnitudes in the range of $0.2-0.7 \mathrm{~dB}$. Four perturbations had positive sign while two were negative The VLF perturbation accompanying the elve event marked by ' 1 ' in Fig. 4 was seen on the HWV $(18.3 \mathrm{kHz})$ transmitter signal, those associated with the elve numbered ' 2 ' were observed on the HWV $(18.3 \mathrm{kHz})$, NAA $(24.0 \mathrm{kHz})$, and NRK $(37.5 \mathrm{kHz})-$ Crete GCPs, while those occurring simultaneously with the elve ' 3 ' were identified in the HWV $(18.3 \mathrm{kHz})$ and NRK $(37.5 \mathrm{kHz})$ amplitude recordings. It is an interesting observational fact that the elve number ' 3 ' related to VLF perturbations seen on relatively remote ( $\sim 400 \mathrm{~km})$ GCPs which seems to be in disagreement with theory since it would imply wide angle scattering. This contradiction could be resolved in two ways. One would be to suppose that the ionised region associated with the elve has about the same size as the region emitting light $(\sim 250 \mathrm{~km})$ in 

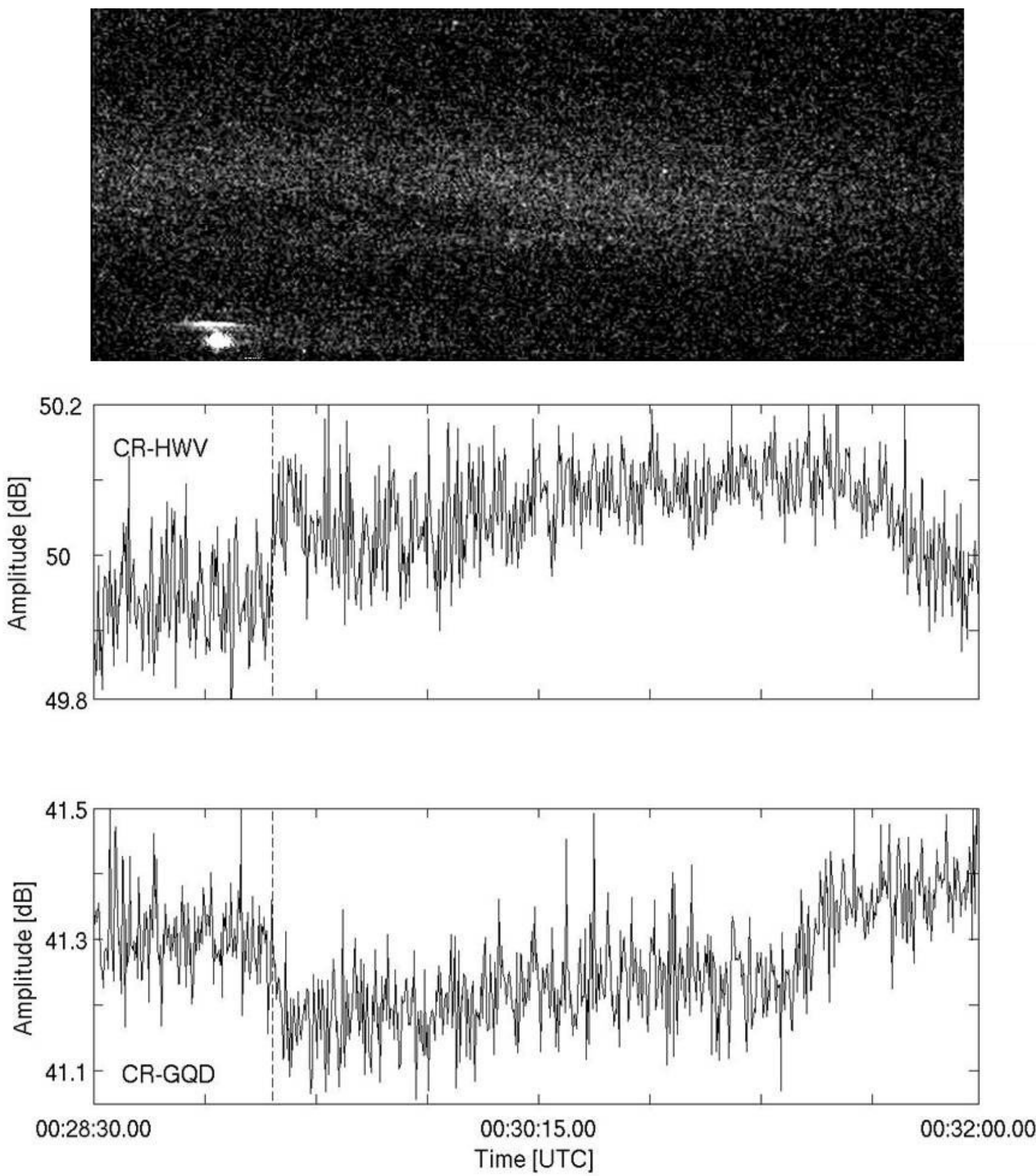

Fig. 3. Early VLF perturbations observed on the Crete - HWV (middle panel) and Crete-GQD (bottom panel) GCPs in association with the elve captured on 24 July 2003 at 00:29:12.757 UTC (top panel). The vertical dashed lines mark the occurrence of the elve.

which case the scattering can be assumed to be narrow angle. The other possibility is that there is a sprite occurring below the elve which acounts for the wide angle scattering of the incident signal. 


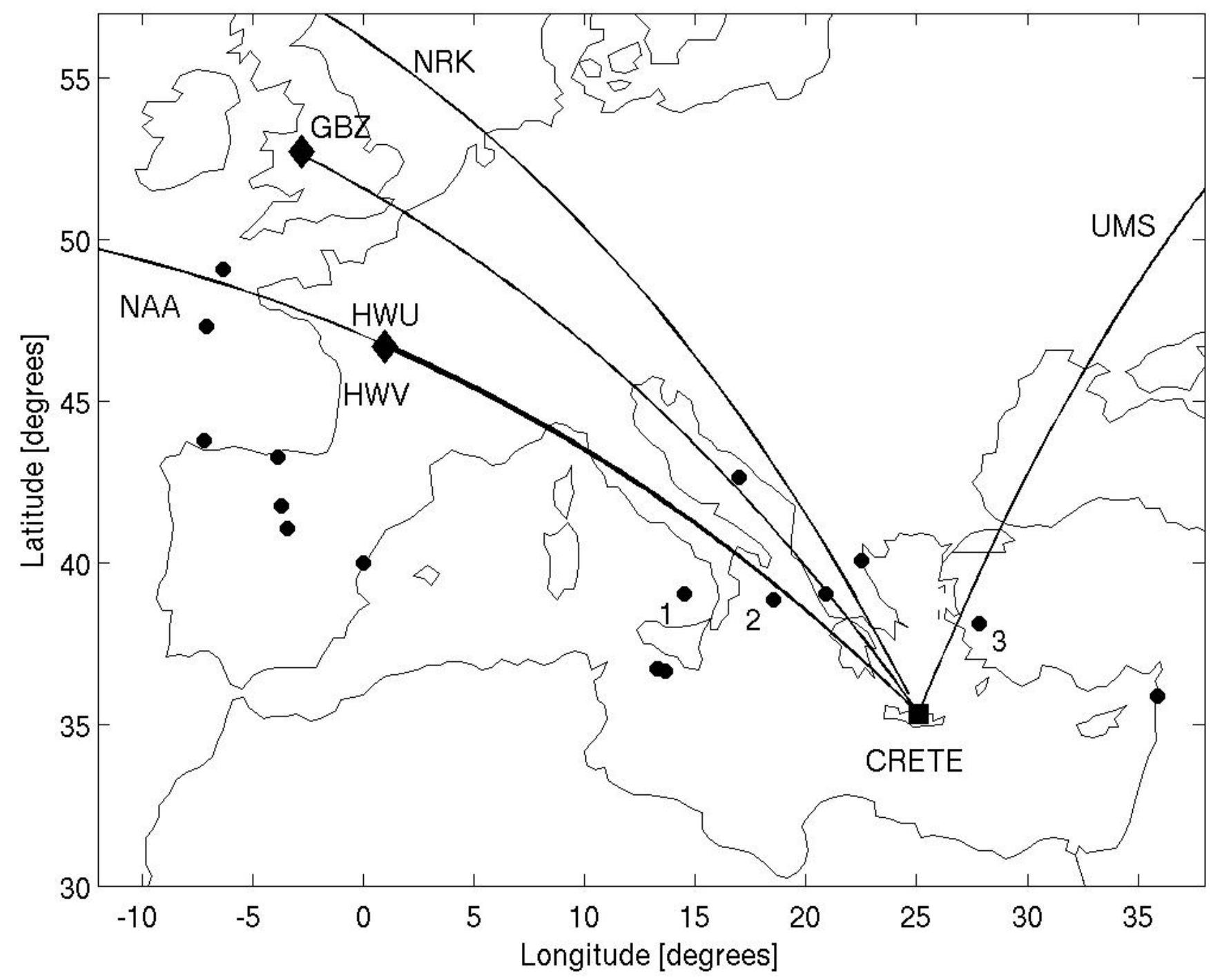

Fig. 4. Locations of the elves observed by ISUAL over Europe (circles) during the October, 2004 - March, 2005 period, along with the GCPs from the VLF transmitters to the Crete receiver. The numbers mark the events accompanied by early VLF perturbations.

A typical example of an early/fast perturbation (observed on the $18.3 \mathrm{kHz}$ signal in association with the elve marked by ' 1 ' in Fig. 4) and the corresponding optical image are presented in Fig. 5, with the dashed line marking the occurrence of the elve. All three early VLF perturbations seen in the $18.3 \mathrm{kHz}$ transmitter signal had relaxation times of $\sim 2 \mathrm{~min}$.

The VLF events identified in the 24.0 and $37.5 \mathrm{kHz}$ signals had a slower relaxation which was eventually masked by other variations in the signal level.

Between July, 2004 and July, 2005, ISUAL captured 282 elves over North America. VLF data covering most of the region of the elve observations was supplied by eight receivers, four of them being part of the Stanford HAIL (Holographic Array for Ionospheric and Lightning research) network (Cheyenne, Boulder, Parker and Las Vegas), while the other four being the Taylor, Boston, Arecibo receiver sites and Palmer station at Antarctica. The VLF receiver specifications are identical to those of the Crete station. Amplitude and phase time series from the NPM $(21.4 \mathrm{kHz}$, Lualualei, Hawaii), NAA $(24.0 \mathrm{kHz}$, Cutler, Maine), NLK (24.8 kHz, Arlington, Washington), and NAU $(40.75 \mathrm{kHz}$, Aguada, Puerto Rico) VLF transmitters were recorded daily from 01:00 to 13:00 UTC, except for the Palmer station which was operating synoptically.

VLF data was only available for 51 elves, in most cases for multiple GCPs. 42 out of the 51 elves were situated at lateral distances less than $500 \mathrm{~km}$ from the nearest GCP, whereas the distances from the elves to the receivers were $>2000 \mathrm{~km}$, that is, much larger than those found previously for the European sector. In Fig. 6 the geographic locations of the elve- 

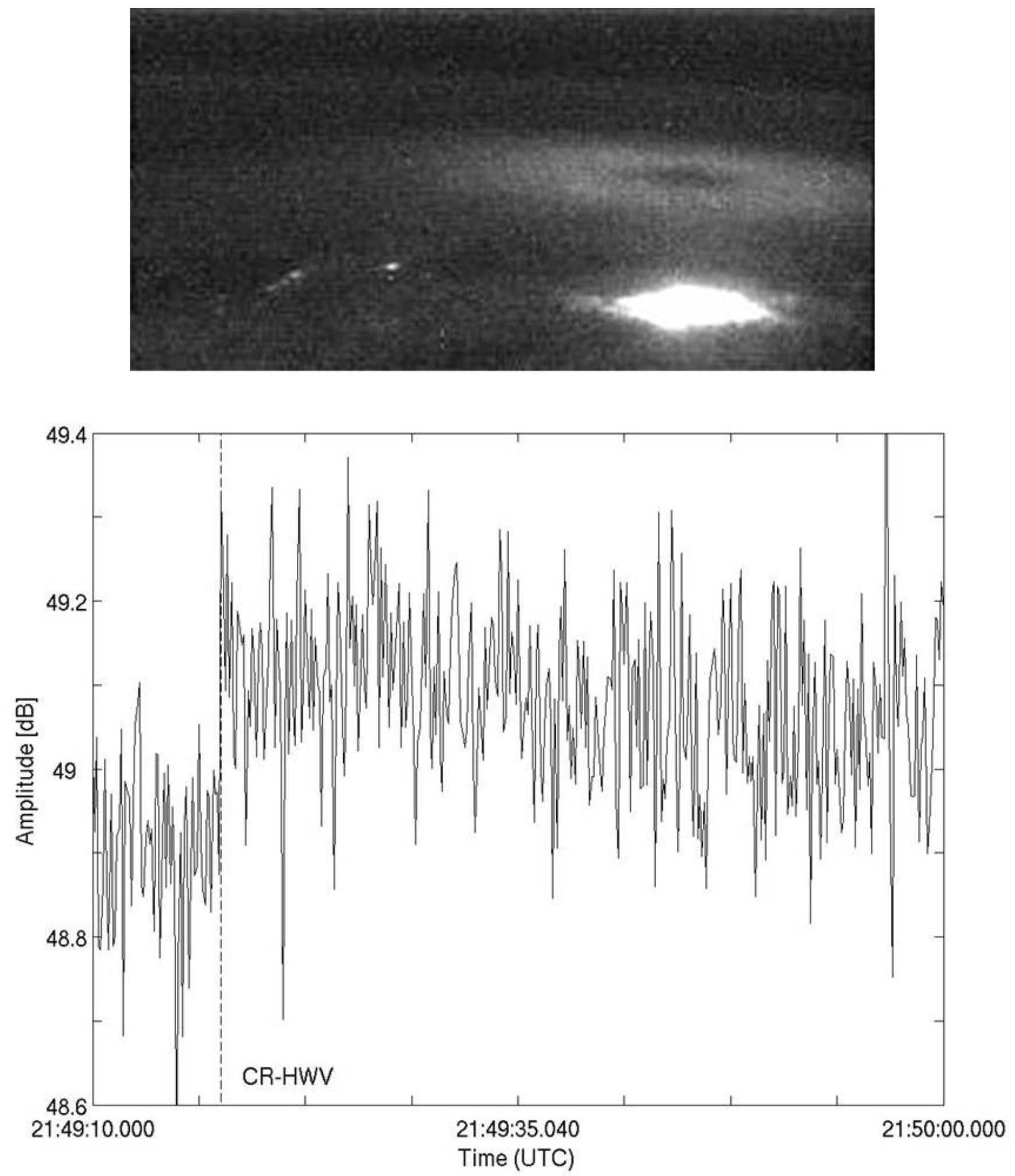

Fig. 5. Optical image of the elve (top panel) marked by ' 1 ' in Fig. 4 and the associated early/fast VLF perturbation as seen in the HWV -Crete amplitude time series (bottom panel). 


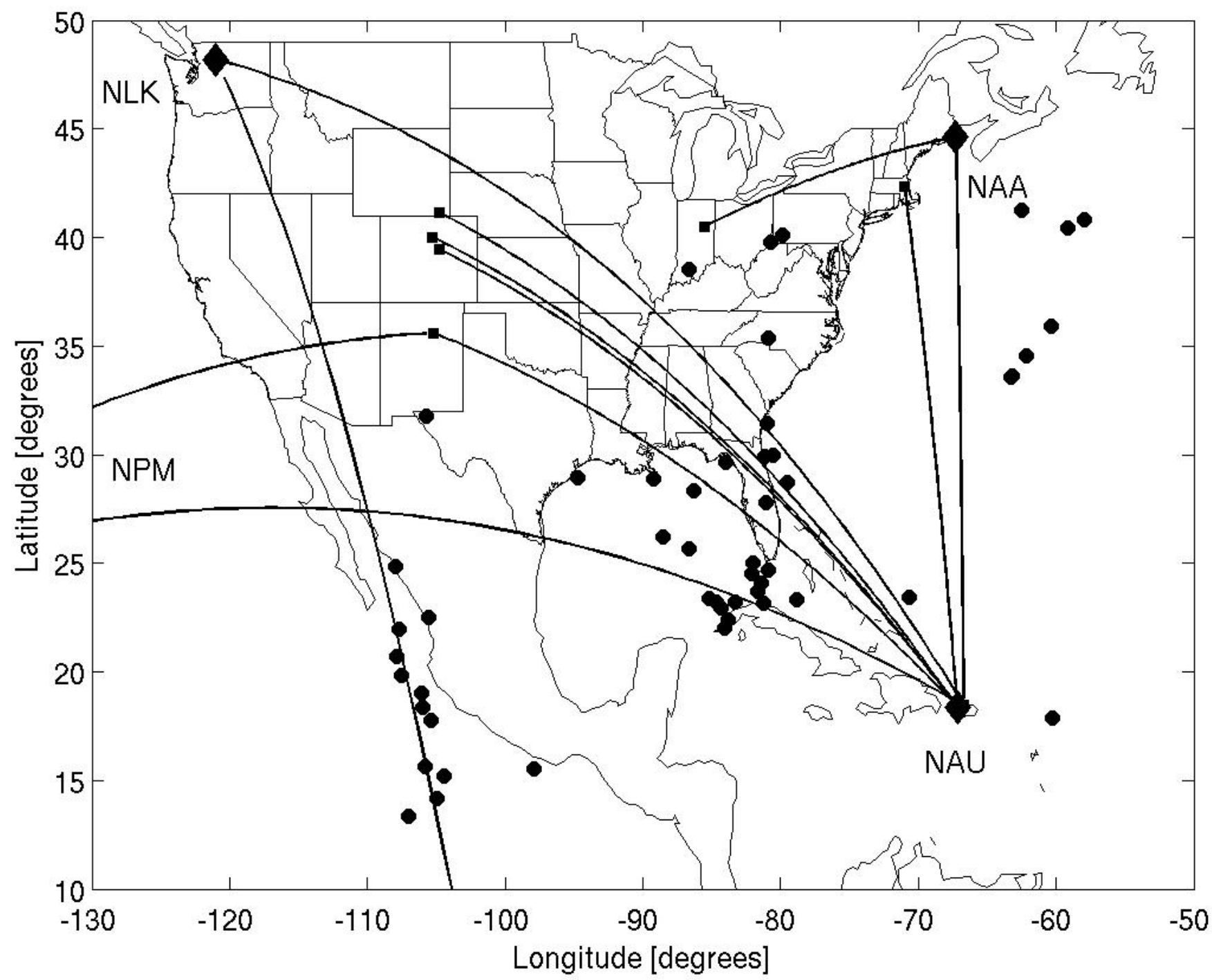

Fig. 6. Locations of the elves observed over the American continents during the period July 2004 - July 2005 (circles). Solid lines represent the GCPs from the VLF transmitters (rhomboids) to the receiver sites (squares) considered in the present analysis.

causative discharges along with the HAIL receiver sites and the GCPs involved in the analysis are presented. The amplitude and phase time series were thoroughly analysed for each receiver-transmitter combination, but no perturbations were found in association with the elves.

\section{Summary and concluding comments}

The existence of perturbations of sub-ionospheric VLF transmissions associated with elves has been investigated. VLF data recorded at various receiver sites were analysed in relation to optical elve observations made from the ground in Europe during the EuroSprite2003 campaign and from space by ISUAL on board the FORMOSAT-2 satellite over the European and American continents.
All five elves captured during EuroSprite2003 were accompanied by VLF perturbations. The perturbation magnitudes were in the range of $0.15-0.4 \mathrm{~dB}$ and the relaxation times were $\sim 2-3 \mathrm{~min}$. Three elves were initiated by strong positive cloud-to-ground discharges with peak currents exceeding $100 \mathrm{kA}$. The lightning detection network failed to identify the causative discharges for two of the elves. In both these cases, however, broadband VLF recordings revealed strong sferics coincident with the elve occurrences.

Finally, the relaxation times of the perturbations seen on the $18.3 \mathrm{kHz}$ link were much shorter than those identified in the 24.0 and $37.5 \mathrm{kHz}$ signals, suggesting that the higher frequency waves, which reflect at higher altitudes, can detect the upper, and thus longer-lived, regions of ionisation changes anticipated during elve events. 
ISUAL observed 17 elves over Europe. Three of them were accompanied by early/fast VLF perturbations seen on multiple transmitter-receiver GCPs, having magnitudes of $0.2-0.7 \mathrm{~dB}$. The relaxation times of the early VLF perturbations identified in the $18.3 \mathrm{kHz}$ signal were $\sim 2 \mathrm{~min}$, while those seen in the 24.0 and $37.5 \mathrm{kHz}$ time series had longer recoveries which could not be measured exactly due to other changes in the signal level.

There were no VLF perturbations accompanying the elves observed by ISUAL over North America, which, however, have occurred at rather large distances from the receivers.

The occasional existence of early VLF perturbations accompanying elves is a clear proof of electron density enhancements/depletions caused by the lightning EMP leading to a sharpening of the electron density profile in the lower ionosphere. Ionisation changes during elve events have already been predicted by modelling studies several years ago (e.g., Taranenko et al., 1993; Inan et al., 1996a). The only experimental evidence on elve-related ionisation has been inferred for the first time recently by Mende et al. (2005), using photometric observations made by ISUAL.

The observations presented here show that the ionisation changes caused by the lightning EMP can occasionally extend below the VLF reflection height and thus can become detectable via VLF remote sensing. However, the possibility of detection seems to be highly sensitive on the distance of the elve from the GCP and the receiver. It is interesting that no VLF perturbations were observed in relation to the numerous elves captured over North America. One possible reason can be that these events were located at distances $>2000 \mathrm{~km}$ from the receivers, while those observed over Europe were much closer. This fact suggests that propagation effects may play an important role. Theory and numerical calculations predict strong inter-modal scattering between dominant TM modes upon incidence on lower ionospheric ionisation enhancements (e.g. see Nunn, 1997). These modes can be attenuated over the long propagation distances to the receiver and thus the VLF perturbations can be masked by noise. However, modelling of the perturbations would be highly desirable to clarify this issue. Another reason can be the absence of ionisation during elves in cases when the EMP intensity is enough to excite optical emissions (the electron energy threshold is $7.0 \mathrm{eV}$ ), but it is insufficient to produce ionisation due to the higher energies $(15.6 \mathrm{eV})$ needed for this process.

Acknowledgements. Á. Mika and R. J. Steiner acknowledge the financial support given by the European Community's Human Potential Programme under contract HPRN-CT-2002-00216. The Stanford contribution to this work was supported by a Stanford Graduate Fellowship and ONR Grant N00014-03-1-0333. The authors would like to thank F. Lefeuvre, M. Parrot and LPCE for providing the Nançay broadband data and S. Pédeboy from Météorage for lightning data. Á. Mika would like to express her gratitude to J. Brundell for helpful discussions and to Oscar van der Velde for identifying additional elves in the EuroSprite2003 database and for providing information on their azimuths.

Topical Editor M. Pinnock thanks J. Lichtenberger and another Referee for their help in evaluating this paper.

\section{References}

Barrington-Leigh, C. P. and Inan, U. S.: Elves triggered by positive and negative lightning discharges, Geophys. Res. Lett., 26, 683686, 1999.

Chern, J. L., Hsu, R. R., Su, H. T., Mende, S. B., Fukunishi, H., Takahashi, Y., and Lee, L. C.: Global survey of upper atmospheric transient luminous events on the ROCSAT-2 satellite, J. Atmos. Sol.-Terr. Phys., 65, 647-659, 2003.

Dowden, R. L.: Comment on "VLF signatures of ionospheric disturbances associated with sprites" by Inan et al., Geophys. Res. Lett., 23, 3421-3422, 1996.

Dowden, R. L., Brundell, J., Lyons, W. A., and Nelson, T.: VLF scattering from sprites and elves, Paper presented at the US Fall AGU meeting, 1995.

Dowden, R. L., Brundell, J. B., Lyons, W. A., and Nelson, T.: Detection and location of red sprites by VLF scattering of subionospheric transmissions, Geophys. Res. Lett., 23, 17371740, 1996.

Fukunishi, H., Takahashi, Y., Kubota, M., Sakanoi, K., Inan, U. S., and Lyons, W. A.: Elves: Lightning-induced transient luminous events in the lower ionosphere, Geophys. Res. Lett., 23, 21572160, 1996.

Haldoupis, C., Neubert, T., Inan, U. S., Mika, A., Allin, T. H., and Marshall, R. A.: Subionospheric early VLF signal perturbations observed in one-to-one association with sprites, J. Geophys. Res., 109, A10303, 2004.

Hobara, Y., Iwasaki, N., Hayashida, T., Hayakawa, M., Ohta, K., and Fukunishi, H.: Interrelation between ELF transients and ionospheric disturbances in association with sprites and elves, Geophys. Res. Lett., 28, 935-938, 2001.

Hobara, Y., Hayakawa, M., Ohta, K., and Fukunishi, H.: Lightning discharges in association with mesospheric optical phenomena in Japan and their effect on the lower ionosphere, Adv. Polar Upper Atmos. Res., 17, 30-47, 2003.

Inan, U. S., Shafer, D. C., and Yip, W. Y.: Subionospheric VLF Signatures of Nighttime D Region Perturbations in the Vicinity of Lightning Discharges, J. Geophys. Res., 93, 11455-11472, 1988.

Inan, U. S., Rodriguez, J. V., and Idone, V. P.: VLF signatures of lightning-induced heating and ionization of nighttime D region, Geophys. Res. Lett., 20, 2355-2358, 1993.

Inan, U. S., Sampson, W. A., and Taranenko, Y. N.: Space-time structure of optical flashes and ionization changes produced by lightning-EMP, Geophys. Res. Lett., 23, 133-136, 1996a.

Inan, U. S., Slingeland, A., Pasko, V. P., and Rodriguez, J. V.: VLF and LF signatures of mesospheric/lower ionospheric response to lightning discharges, J. Geophys. Res., 101, 5219-5238, 1996 b.

Mende, S. B., Frey, H. U., Hsu, R. R., Su, H. T., Chen, A. B., Lee, L. C., Sentman, D. D., Takahashi, Y., and Fukunishi, H.: D region ionization by lightning-induced electromagnetic pulses, J. Geophys. Res., 110, a11312, 2005.

Mika, Á., Haldoupis, C., Marshall, R. A., Neubert, T., and Inan, U. S.: Subionospheric VLF signatures and their association with 
sprites observed during EuroSprite-2003, J. Atmos. Sol.-Terr. Phys., 67, 1580-1597, 2005.

Morel, C. and Sénési, S.: SAF NWC scientific report for MTR on PGE11 rapid developing thunderstorms, METEO FRANCE CNRM, http://www.meteorologie.eu.org/RDT/doc/ SAF-NWC-MF_CNRM-SCI-MTR-001.pdf, 2000.

Neubert, T., Allin, T. H., Blanc, E., Farges, T., Haldoupis, C., Mika, A., Soula, S., Knutsson, L., van der Velde, O., Marshall, R. A., Inan, U., Sátori, G., Bór, J., Hughes, A., Collier, A., Laursen, S., and Rasmussen, I. L.: Co-ordinated observations of transient luminous events during the EuroSprite2003 campaign, J. Atmos. Sol.-Terr. Phys., 67, 807-820, 2005.

Nunn, D.: On the numerical modelling of the VLF Trimpi effect, J. Atmos. Sol.-Terr. Phys., 59, 537-560, 1997.

Reising, S. C., Inan, U. S., and Bell, T. F.: ELF/VLF radio atmoshperic signatures of lightning flashes associated with sprites, EOS, 76, 105, 1995.

Rodger, C. J.: Red sprites, upward lightning, and VLF perturbations, Rev. Geophys., 37, 317-336, 1999.

Rodger, C. J.: Subionospheric VLF perturbations associated with lightning discharges, J. Atmos. Sol.-Terr. Phys., 65, 591-606, 2003.

Rodger, C. J., Cho, M., Clilverd, M. A., and Rycroft, M. J.: Lower ionospheric modification by lighting-EMP: Simulation of the night ionosphere over the United States, Geophys. Res. Lett., 28, 199-202, 2001.
Rowland, H. L.: Theories and simulations of elves, sprites and blue jets, J. Atmos. Sol.-Terr. Phys., 60, 831-844, 1998.

Rowland, H. L., Fernsler, R. F., and Bernhardt, P. A.: Breakdown of the neutral atmoshpere in the $\mathrm{D}$ region due to lightning driven electromagnetic pulses, J. Geophys. Res., 101, 7935-7945, 1996.

Strangeways, H. J.: Lightning, Trimpis, and Sprites, Rev. Radio Sci.1993-1996, pp. 741-780, 1996.

Sukhorukov, A. I., Rudenchik, E. A., and Stubbe, P.: Simulation of the strong lightning pulse penetration into the lower ionosphere, Geophys. Res. Lett., 23, 2911-2914, 1996.

Takahashi, Y., Kubota, M., Sakanoi, K., Fukunishi, H., Inan, U. S., and Lyons, W. A.: Spatial and temporal relationship between lower ionospheric flashes and sprites, EOS, 76, 106, 1995.

Taranenko, Y. N.: Interaction with the lower ionosphere of electromagnetic pulses from lightning: heating, attachment, ionization, and optical emissions, Ph.D. thesis, Stanford University, 1993.

Taranenko, Y. N., Inan, U. S., and Bell, T. F.: Interaction with the lower ionosphere of electromagnetic pulses from lightning: heating, attachement, and ionization, Geophys. Res. Lett., 20, 15391542, 1993. 